pag

Business School

WORKING PAPER SERIES

Working Paper $2014-264$
Stratégie de "tourisme durable": Validation empirique dans le Groupe TUI en Tunisie

Sandra Camus

Lubica Hikkerova

Soumaya Hergli

Guillaume Marceau

Jean-Michel Sahut

http://www.ipag.fr/fr/accueil/la-recherche/publications-WP.html

IPAG Business School

184, Boulevard Saint-Germain

75006 Paris

France 


\title{
Stratégie de "tourisme durable": Validation empirique dans le Groupe TUI en Tunisie
}

\author{
Sandra Camus ${ }^{\mathrm{a}}$, Lubica Hikkerova ${ }^{\mathrm{b}}$, Soumaya Hergli ${ }^{\mathrm{c}}$, Guillaume Marceau ${ }^{\mathrm{d}}$ \\ Jean-Michel Sahut ${ }^{\mathrm{e}}$
}

\author{
${ }^{a}$ Associate Professor, LUNAM, University of Angers, GRANEM - UMR 49, France \\ ${ }^{b}$ Assistant Professor, IPAG Business School - Paris, France \\ ${ }^{c}$ PhD Student, CEREGE EA 1722, University of Poitiers, France \\ ${ }^{d}$ Associate Professor, CERGAM-University of Aix Marseille, France \\ ${ }^{e}$ Professor, IPAG Business School - Paris, France \\ \& CEREGE EA 1722, University of Poitiers, France
}

\section{RESUME}

Cet article étudie le Groupe TUl et sa chaîne hôtelière Magic Life. Les succès remarquables de ce Groupe en matière de développement durable l'ont amené à développer ses propres normes et standards. Malheureusement, les politiques publiques et les initiatives privées comme celle de TUI sont aujourd'hui entravées par les réticences des entreprises locales à l'égard des projets en cours. Ceci montre qu'une démarche de développement "de tourisme durable" doit pouvoir s'appuyer sur I'ensemble des parties prenantes (Gouvernement, entreprises, citoyens, groupe d'intérêts) pour être véritablement efficace.

\section{ABSTRACT}

This article examines the company TUI and its Magic Life hotel chain. The remarkable success of this group in terms of sustainable development led him to develop its own norms and standards. Unfortunately, public policies and private initiatives such as TUI are now hampered by the reluctance of local businesses against projects. This shows that a development approach "sustainable tourism" should be supported by all stakeholders (government, businesses, citizens, interest group) to be truly effective.

Post Print : Magheb-Machrek, n²16, 2013. 
Située à la jonction des deux bassins oriental et occidental de la Méditerranée, la Tunisie occupe une position géographique stratégique présentant un potentiel naturel propice au tourisme avec ses merveilleuses plages, un paysage très diversifié, un patrimoine culturel important, un climat dans l'ensemble doux et ensoleillé... Cette richesse constitue un avantage compétitif pour le développement de l'activité touristique qui représente l'un des secteurs les plus dynamiques avec 7\% du PIB générant chaque année entre 18 et $20 \%$ des recettes en devise et employant plus de 400000 personnes ${ }^{1}$.

Ce développement du tourisme se traduit également par l'augmentation des impacts négatifs environnementaux tels que : la dégradation du littoral (suite à l'urbanisation croissante dans les agglomérations côtières), l'épuisement des ressources naturelles en eau, sol, énergie..., la pollution (en particulier due aux eaux usées, l'augmentation du trafic routier), l'érosion des dunes, la modification des écosystèmes terrestres et marins, l'endommagement des barrières de corail..., des impacts socioculturels importants qui se traduisent, entre autres, par une modification ou une perte des valeurs et des identités indigènes, par des problèmes éthiques (le travail et l'exploitation des enfants par exemple)..., des déséquilibres économiques puisqu'il bénéfice principalement aux régions côtières et qu'il profite peu à la population du pays hôte (Hergli et al., 2012)...

Pour faire face à ces impacts négatifs et améliorer son rôle positif, le secteur touristique en Tunisie se tourne vers le «tourisme durable » selon une démarche de développement local, intégrant, d'une part, l'éducation environnementale et, d'autre part, l'animation sociale et économique selon un guide de bonnes pratiques comme le signale N. Le Scouarnec ${ }^{2}$ : «Tout autant que les autres secteurs, le tourisme est appelé à produire un guide de bonnes pratiques et à s'y conformer afin de participer à la réalisation du développement durable...

L'objectif de cet article est de montrer comment la Tunisie a initié une stratégie de tourisme durable, et les différents freins à sa mise en œuvre. Nous analyserons en particulier le cas du groupe TUI au travers de sa chaine Magic Life, pionnière dans le domaine, afin de dresser des perspectives d'avenir.

\section{ANALYSE DU CONTEXTE TUNISIEN}

A partir les années 90, une nouvelle stratégie économique a été adoptée par la Tunisie dans un cadre de «développement durable et de partenariat », suite à la conférence de Rio (1992) et la déclaration de Barcelone (1995). Ainsi, une attention particulière a été accordée au développement touristique par l'instauration des plans de mise à niveau (MAN) dans une logique de «développement durable » garantissant sur le long terme la durabilité de ce secteur par «la recherche du juste équilibre entre ses aspects environnementaux, socioculturels et économiques, dans une perspective de viabilité à long terme»(PNUE 2006) $)^{3}$. En effet, le

\footnotetext{
${ }^{1}$ UNWTO Annual Report 2010.

${ }^{2}$ Chef du bureau, des relations internationales du département du tourisme en France ; selon une déclaration à la presse, Un guide de bonnes pratiques pour réaliser le développement durable, au 14/08/2009,

${ }^{3}$ Joëlle Paquet (2010), Rapport évolutif : L'application des principes du développement durable: Le cas du tourisme ; LEPPM (Laboratoire d'étude sur les politiques publiques et la mondialisation).
} 
tourisme durable doit, parvenir à une allocation optimale des ressources et établir un équilibre adéquat entre ses trois piliers. L'aspect environnemental représente la plaque tournante du développement durable du tourisme laquelle exige une exploitation optimale des ressources de l'environnement tout en préservant les processus écologiques essentiels et en aidant à sauvegarder les ressources naturelles. Le deuxième pilier doit garantir la durabilité de l'aspect socioculturel, en préservant le patrimoine culturel des communautés d'accueil. Enfin, le pilier économique vise à assurer une activité économique viable sur le long terme, c'est-à-dire offrant à toutes les parties prenantes des avantages socio-économiques équitablement répartis, notamment des emplois stables, des perspectives de profit pour les entreprises et des services sociaux pour les communautés d'accueil, qui contribuent ainsi à la réduction de la pauvreté.

\section{Sur le plan environnemental}

La concentration de l'activité touristique sur le littoral (85\% de la capacité hôtelière et $92 \%$ des nuitées ${ }^{4}$ ), constitue un vecteur de dynamisme mais entraîne de sérieux problèmes, notamment : la congestion et la dégradation de l'environnement et des espaces naturels. Cette dégradation constitue une menace réelle face au développement touristique et un risque de non durabilité qui affecte la compétitivité touristique à long terme. La dégradation de la qualité de l'eau de baignade représente également un autre phénomène auquel le tourisme tunisien devra faire face. En particulier, suite à la construction du port de plaisance Marina de Yasmine - Hammamet, un phénomène de cumul d'algues, est apparu sur les plages et dans l'eau, engendrant une dégradation de la qualité de l'eau et de l'environnement général. Suite à cette situation certains tours opérateurs ont annulé des contrats. Il est important aussi de signaler que la consommation de l'eau et de l'énergie reste croissante au fil du temps. En effet, la rareté des ressources en eau et en énergie de la Tunisie et la pression du secteur touristique sur ces ressources pourraient affecter le développement des secteurs économiques grands consommateurs de ces ressources tel que le tourisme.

De plus, les changements climatiques, dus notamment à l'émission de plus en plus importante de gaz à effets de serre suite à l'augmentation des déplacements aériens et terrestres ${ }^{5}$, représentent un risque de non durabilité du secteur touristique avec l'érosion marine qui s'accentue par l'élévation du niveau de la mer, l'élévation de la température qui affecte les activités touristiques pratiquées par les visiteurs ...

La «durabilité » environnementale de ce secteur recommande ainsi de «développer et promouvoir un tourisme "respectueux de l'environnement » permettant une utilisation dans une logique «durable » des ressources en eau, énergie et sol, ainsi qu'une protection du littoral $^{6}$. Cela nécessite la mise en place de mécanismes de régulation et d'utilisation rationnelle des ressources naturelles et de lutte contre toutes les formes de pollution, mais également de promouvoir d'autre formes que le tourisme de masse classique comme l'écotourisme...

\section{Sur le plan socioculturel}

\footnotetext{
${ }^{4}$ Ministère de l'environnement et de développement durable (MEDD), l'agence nationale de l'environnement et de protection de l'environnement (ANPE), 2010, guide pour le tourisme durable en Tunisie.

${ }^{5}$ Selon l'OMT 2007 : "La croissance annuelle de 4\% des arrivées touristiques impliquerait une croissance des émissions de $\mathrm{CO} 2$ de l'ordre de $152 \%$ d'ici 2035 (selon le rapport MEDD et ANPE 2010).

${ }^{6}$ Suite à la forte pression exercée par le tourisme et l'urbanisation liée au tourisme sur le littoral (rapport des Nations Unies et Plan Bleu de 2005), l'Agence de Protection et d'Aménagement du Littoral a été créée pour la gestion et la protection de ce dernier qui représente la ressource principale du tourisme tunisien.
} 
L'implantation des hôtels était en «zoning », éloignée des villes et de la population locale provoquant ainsi, une ségrégation sociale. Aujourd'hui, le concept même du tourisme change et ce modèle de juxtaposition des hôtels de quelques centaines de lits dans des zones désertes est devenu obsolète. En effet, le tourisme n'est pas seulement une hôtellerie se basant essentiellement sur une production d'hôtels et de zones touristiques, c'est plutôt un "projet touristique pour la société et pas nécessairement pour le promoteur touristique ». Le touriste d'aujourd'hui est intéressé plutôt par des résidences, des apparts-hôtels, des hôtels de charme mais aussi des lieux de loisir et d'animation, une mise en tourisme de la ville en tant que composante essentielle du tourisme afin que le touriste ne s'ennuie pas en optant pour la Tunisie» (Chaieb, 2009).

La durabilité sociale se limite souvent aux aspects d'équité et de lutte contre l'exclusion sociale (Parra et Moulaert, 2011). La durabilité culturelle invite les touristes et la population locale à profiter des nombreux sites d'une grande richesse culturelle. De ce fait, la durabilité socioculturelle recommande de «développer et de promouvoir un tourisme intégré» permettant de sensibiliser toutes les parties prenantes et les impliquer dans une logique «durable » afin d'améliorer la compétitivité du secteur par l'amélioration de : l'attractivité de la destination, de la qualité du produit et des recettes touristiques (Chapoutot, 2008).

D'autre part, cette logique «durable » va permettre de se prémunir contre le risque de la dépendance de l'économie régionale du tourisme ${ }^{7}$ par le développement ${ }^{8}$ et la diversification du produit à l'intérieur du pays afin de stimuler la création de l'emploi touristique, l'étalement de la saison et faire face à son caractère instable et précaire.

\section{Sur le plan économique}

Jusqu'à une vingtaine d'années, le souci de la Tunisie était de promouvoir le tourisme par l'implantation massives d'unités hôtelières, sans pour autant donner importance à la diversification. Ce qui s'est traduit par un certain retard en termes de diversité de l'offre par rapport aux autres pays concurrents. Depuis, la Tunisie essaye de développer une mobilité touristico-patrimoniale permettant de faire évoluer l'offre balnéaire et de diversifier le produit touristique, mais ce dernier reste concentré essentiellement sur le littoral (Chapoutot, 2008).

Cette concentration constitue un vecteur de dynamisme mais entraîne de sérieux problèmes, notamment une répartition régionale déséquilibrée, une fluctuation des indicateurs de performance (recettes, emploi...), des effets d'entraînement cycliques sur les autres secteurs...Cela représente un risque de non durabilité économique en termes d'attractivité et de positionnement stratégique.

La «durabilité » économique de ce secteur recommande de «développer et de promouvoir un tourisme diversifie ${ }^{9}{ }^{9}$, déconcentré sur les zones balnéaires et ouvert à l'intérieur du pays dans l'objectif d'étaler la saison touristique, d'élargir la clientèle, notamment en accordant un intérêt particulier aux marchés arabes et lointains, et d'accroître les sources de revenus. Cela

\footnotetext{
${ }^{7}$ Une analyse régionale de la part des emplois dans l'emploi total montre une forte contribution du tourisme (selon le rapport de MEDD et l'ANPE du 2010).

${ }^{8}$ Il est prioritaire de démontrer que le développement touristique se fait avec la participation locale des agents et des produits (Agir ensemble pour un tourisme durable, un guide pour informer, sensibiliser, encourager et passer à l'action, comité 21).

${ }^{9}$ Suite aux recommandations de la conférence de Rio (1992), de la commission nationale pour le développement durable (décret 93-2061) et de l'atelier de travail Agenda 21.
} 
nécessité de diversifier les modes d'hébergement (hôtels de charme, Appart hôtels...) et de restauration, mais également les activités liés aux sports terrestres et pas seulement nautiques (comme le golf compte tenu de sa capacité à attirer certaines catégories de clientèles aisées), à la santé (thalassothérapie et balnéothérapie notamment), à la culture (excursions par exemple), aux congrès, séminaires, salons, foires, et expositions...

\section{ASPECTS INSTITUTIONNELS DU "TOURISME DURABLE" EN TUNISIE}

La mise en œuvre d'une démarche de tourisme durable est un processus complexe et nécessite la mise en place d'une véritable gestion de programmes transverses impliquant toutes les parties prenantes dans un modèle «durable». Ainsi, une collaboration cohérente et pertinente entre ces acteurs, à tous les échelons: local, régional, national et international est nécessaire afin de bénéficier des effets de synergies et des économies d'échelle. Il est évident que toute démarche de progrès ou de développement est positive si elle est validée jusqu'au bout de la chaine et si on la fait vivre dans le temps ${ }^{10}$. Afin de donner une certaine visibilité à ce type de démarche de tourisme durable, les organisations cherchent à les faire valider par l'obtention des normes, certificats, labels...

Un label «approche produit » est un signe de qualité, d'application volontaire, garantissant qu'un produit respecte des critères environnementaux et/ou sociaux. Aujourd'hui, le bon produit n'est plus celui qui est le moins cher mais plutôt celui qui est plus respectueux de l'environnement, de l'homme, de l'hygiène...Un label "approche site" (ou entreprise) s'intéresse à l'organisation des organismes ${ }^{11}$. Une certification fait référence à un constat de conformité d'un produit vis-à-vis de certaines normes et critères (Cromer, 2011). Les normes sont adoptées au sein d'organismes officiels (Afnor en France, ISO au niveau international) selon une procédure formelle précise. Tandis que les labels peuvent renvoyer aux normes pour certaines de leurs exigences ou encore suivre des méthodologies normalisées pour l'élaboration de leurs cahiers des charges et leur contrôle. A l'extrême, les labels peuvent constituer des certifications au sens de $1^{\prime}$ ISO $^{12}$ lorsqu'ils élaborent un cahier de charge reposant sur un organisme compétent et impartial.

Pour les clients, on observe qu'il est nécessaire aujourd'hui que les offres touristiques dites durables crédibilisent leur approche par un label. En effet, selon les différentes études commanditées par voyages-sncf.com, $87 \%$ des voyageurs sont prêts à faire confiance à un label garantissant que leur voyage respecte les principes du tourisme responsable. Selon une autre étude d'Atout France - GMV Conseil, 80\% des Français interrogés considèrent la présence d'un label comme une garantie pour la « démarche tourisme durable ». Il est ajouté que l'existence d'un contrôle extérieur réalisé par un organisme indépendant et reconnu est demandée par 55\% des personnes interrogées. L'échelon doit être à minima européen ou, encore mieux, international (Cromer, 2011).

\footnotetext{
${ }^{10}$ Ruffier.N, 2008, tourisme durable : le label ATR, un passage obligé pour les TO d'aventure ? Le quotidien du tourisme

${ }^{11}$ Czaryski.S, 2009, abcvert, labels et consommation durable.

12 Procédure par laquelle une tierce partie donne une assurance écrite qu'un produit ou un processus est conforme aux exigences spécifiées » (Guide ISO/CEI 2:2004).
} 
Dans ce cadre d'analyse, la Tunisie a mis en place plusieurs programmes nationaux ou en partenariat avec des organismes étrangers, dans l'objectif de présenter une offre touristique inscrite dans une logique «Durable » permettant l'obtention de normes, certificats, labels...

On peut citer par exemple le programme tuniso-allemand pour l'environnement visant depuis 2003 la prévention et la lutte contre la pollution et aussi la coopération technologique, le programme environnement et énergie qui a été initié en partenariat avec l'Union Européenne pour améliorer la compétitivité des entreprises tunisiennes dans les aspects environnementaux et énergétiques, le projet de production propre pour la Tunisie (PPPT) initié en partenariat avec la Suisse et l'ONUDI dans l'objectif de sensibiliser les entreprises à l'efficacité des ressources et la production propre (ERPP) ${ }^{13} \ldots$

On peut ainsi remarquer que la Tunisie a accordé une place prépondérante à l'environnement qui représente une composante déterminante dans la compétitivité du produit touristique. Pour renforcer cette place, et moyennant un cofinancement de la Commission Européenne ainsi que l'assistance de l'agence allemande de coopération technique (GTZ), le ministère de l'environnement et du développement durable a instauré le projet « Ecolabel tunisien ».

«L'Ecolabel » est un concept qui se place au dessus de la norme écologique ISO 14001. «L'écolabel tunisien» va permettre «l'augmentation de la compétitivité des produits tunisiens, la protection de l'environnement, l'incitation à l'utilisation de nouvelles technologies de production plus propres ainsi que des meilleures technologies disponibles de dépollution, le marketing d'une production/consommation plus responsable afin de soutenir un développement durable ${ }^{14}$.

L'écolabel tunisien est un label de performance environnementale de type $\mathrm{I}^{15}$ selon les définitions ISO. C'est un programme volontaire, multicritères, attribué aux produits de plus haut niveau de qualification et qui présentent le cas échéant les plus larges opportunités de durée de vie et qui respectent un ensemble de critères techniques et écologiques dans le cadre tri - dimensionnel du développement durable : environnemental, social et économique ${ }^{16}$. Appliqué aux services d'hébergement, il a pour objectif de mieux les valoriser auprès des consommateurs européens soucieux de l'environnement.

\section{MISE EN GUVRE D'UNE STRATEGIE DE TOURISME DURABLE EN TUNISIE : LE CAS DE MAGIC LIFE}

Magic Life est une chaîne hôtelière autrichienne appartenant au groupe TUI. Elle a ouvert son premier club «All inclusive » en Turquie en 1991. Elle gère actuellement en Tunisie huit clubs offrant une gamme de services complète (transport, hébergement, restauration, divertissement) sur un mode de forfaitaire et avec un positionnement haut de gamme.

Magic Life est la première chaîne en Tunisie ayant obtenu les labels et certificats suivants:

\footnotetext{
${ }^{13}$ webmanagercenter.com - 02 Juin 2009, Enquête d'adhérents.

${ }^{14}$ Ministère de l'environnement et du développement durable, l'écolabel tunisien, pour une production propre et une consommation durable...face aux exigences environnementales du marché international.

${ }^{15}$ Type I est un programme de certification volontaire, multicritères accordé par une tierce partie tenant compte du cycle de vie du produit

${ }^{16}$ La presse, économie verte, Ecolabel Tunisie, clé de voûte de l'exportation, 01/09/2010
} 
- ISO 14001: la plus utilisée de la série des normes ISO 14000; il s'agit d'une norme qui indique les exigences relatives à un système de gestion environnementale, ayant pour but l'élaboration d'une politique en accord avec les exigences réglementaires et la prise en compte des impacts de cette politique sur l'environnement ${ }^{17}$.

- ISO 9001: appartenant à la série des normes ISO 9000 relatives aux systèmes de gestion de la qualité; elle définit des exigences concernant l'organisation d'un système de gestion de la qualité.

- ISO 22000: norme relative à la sécurité des denrées alimentaires.

- Ecolabel Pavillon Bleu18: il s'agit d'un écolabel ayant une forte connotation touristique, attribué aux communes et aux ports de plaisance qui mènent une politique de recherche d'application durable en faveur d'un environnement de bonne qualité.

- Ecolabel Clef verte: label de gestion environnementale pour l'hébergement touristique ${ }^{19}$.

- TUI Ecoresort: label de gestion environnementale initié en interne par le groupe «Ecoresort », ce label de qualité a été créé conjointement par TUI Hôtels \& Resorts et TUI AG Gestion de l'environnement. Les systèmes de gestion environnementale des EcoResorts sont vérifiés par des spécialistes indépendants, accrédités et certifiés selon une norme/ certificat reconnue à l'échelle nationale ou internationale telle que la norme ISO $14001^{20}$.

- TUI Umwelt Champion: label de gestion environnementale initié par le groupe TUI à l'hôtel «Champion » qui a respecté, en se basant sur les réponses de la clientèle aux questionnaires établis chaque année, aux mieux les mesures de l'environnement ${ }^{21}$.

- Ecolabel Tunisien: label de performance environnementale.

Depuis que le premier hôtel "ML Africana Imperial", catégorie 5 étoiles, a obtenu la certification ISO 14001 en 2006, la Direction Générale a décidé de généraliser cette démarche à l'ensemble des hôtels Magic Life. Ainsi, les trois Hôtels ML Africana Impérial, ML Manar à Hammamet et ML Penelope Djerba ont été certifiés ISO 14001, ISO 9001 et ISO 22000.

\section{Enjeux}

Le groupe accorde une place prépondérante à la gestion de l'environnement et considère que «Le respect de l'environnement et des équilibres naturels, aujourd'hui, est la base du développement social, humain et écologique de demain». Depuis 2004, le groupe suit une démarche volontaire «durable » dont les principes sont les suivants:

- adaptation des pratiques de consommation au développement durable du tourisme,

\footnotetext{
${ }^{17}$ CITET, 2005, Etude de faisabilité d'un écolabel Tunisien.

${ }^{18}$ La Tunisie a adhéré depuis 2007 à l'initiative "Pavillon Bleu ». Cet écolabel a été lancé par le MEDD (Ministère de l'environnement et du développement durable) avec le concours de l'ATPNE (Association Tunisienne pour la Protection de la Nature et de l'environnement), certificateur national adhérant à la FEE (Fondation pour l'Education de l'environnement).

${ }^{19}$ CITET, 2005, Etude faisabilité d'un écolabel Tunisien.

${ }^{20}$ http://www.tui-group.com/en/sustainability/env_management/env_awards

${ }^{21}$ http://www.tui-group.com/en/sustainability/env_management/env_awards
} 
- intégration des aspects environnementaux à la stratégie de l'entreprise,

- respect des normes en vigueur en matière de gestion environnementale,

- veille permettant de mesurer et d'améliorer en permanence les performances

environnementales de l'entreprise,

- évaluation et réduction des coûts liés à l'environnement,

- minimisation des risques d'impact de l'activité sur l'environnement,

- développement d'une image en rapport avec la stratégie « durable » de l'entreprise,

- formalisation de la démarche engagée par un label officiel.

\section{Politique de la Direction Générale}

La Direction Générale du Groupe a intégré la stratégie du tourisme durable dans sa stratégie globale et s'est engagée en impliquant toutes les parties prenantes à une amélioration continue du management de la qualité, de l'environnement et de la sécurité.

\section{Objectifs spécifiques à l'alimentaire}

- maitriser la qualité des aliments au niveau des locaux de stockage, de production et de distribution,

- sensibiliser les fournisseurs des matières premières et les responsabiliser quant à

l'application des règles de bonnes pratiques d'hygiène,

- améliorer en permanence la maîtrise des risques liés aux aliments.

\section{Objectifs en matière de Qualité}

- améliorer en permanence le niveau de satisfaction des clients,

- optimiser le budget et réduire le gaspillage,

- améliorer en permanence l'efficacité des processus.

\section{Objectifs spécifiques à l'environnement}

- s'assurer du respect des exigences réglementaires relatives à la protection de

l'environnement,

- réduire et/ou valoriser les déchets,

- rationnaliser la consommation d'eau et d'énergie,

- réduire les risques d'accidents pouvant affecter l'environnement.

La Direction Générale révise les objectifs ci-dessus chaque année afin de s'assurer de leur pertinence, tandis qu'elle s'engage à se doter des moyens nécessaires à leur atteinte.

\section{Stratégie environnementale}

La stratégie environnementale du Groupe se base essentiellement sur les actions suivantes:

- Gestion de l'énergie

- Gestion de l'eau

- Gestion des déchets

- Gestion de la qualité de l'air

- Traitement des eaux

- Gestion du paysage et architecture 
- Sensibilisation des enfants des écoles primaires

- Organisations d'excursions dans des zones protégées

- Célébration des journées internationales et nationales pour l'environnement

- Plantation d'arbres

- Contrôle de la faune

- Gestion des fournisseurs ayant des activités générant des impacts sur l'environnement ${ }^{22}$

Cette démarche a permis de réaliser des économies de $25 \%$ de la consommation d'électricité et de $28 \%$ de la consommation de gaz durant la période allant de 2007 à $2009^{23}$.

\section{Stratégie sociale}

La stratégie sociale se base essentiellement sur les actions suivantes:

- Soutien des organismes sociaux

- Visite des orphelinats et des maisons de retraite

- Création de jardins et de centres sportifs pour le personnel

- Formation continue

Ces mesures sont présentées au personnel lors du recrutement. Il s'agit de proposer à chaque employé des conditions de travail favorables et de le sensibiliser à l'importance de son intégration à une démarche «durable » permettant de réaliser les objectifs de l'entreprise tout en protégeant l'environnement.

Le Groupe s'efforce ainsi de participer activement à la réduction des effets négatifs du tourisme (comme l'exploitation des enfants).

\section{Interprétation}

Le groupe a suivi une stratégie de développement «vivable ${ }^{24}$ » en tenant compte de critères environnementaux et sociaux, tout en proposant une offre touristique et des actions socioculturelles «à visage humain ».

Cette stratégie lui a permis un positionnement de «leader» sur le marché tunisien suite à l'obtention des certificats ISO 14001, 9001, 22000, et des écolabels Pavillon Bleu, Clef Verte, Tui Ecoresort, Tui Umwelt Champion et l'écolabel tunisien.

L'obtention de ces labels correspond effectivement à une réduction des impacts environnementaux et à la limitation de la consommation de ressources naturelles. Elle peut aussi être considérée comme un outil de communication permettant d'attirer une clientèle exigeante en matière d'offre touristique et de respect de l'environnement ${ }^{25}$.

Le groupe a ainsi suivi une démarche exemplaire de «tourisme durable » conciliant le développement économique et l'équilibre écologique, tout en participant localement à

\footnotetext{
${ }^{22}$ Destinations; Développement de stratégies pour un tourisme durable dans les nations méditerranéennes Rapport sur le choix et l'évaluation environnementale des structures pilotes.

${ }^{23}$ Ben Dhafer Z., 2012, journée de lancement du projet de production propre de la Tunisie PPPT.

${ }^{24}$ Conciliant seulement les deux piliers écologique et social sans pour autant attaquer le pilier économique.

${ }^{25}$ Destinations ; Développement de stratégies pour un tourisme durable dans les nations méditerranéennes Rapport sur le choix et l'évaluation environnementale des structures pilotes.
} 
l'ancrage de métiers d'avenir. Agissant sur le territoire tunisien, ce groupe souffre de plusieurs contraintes entravant sa démarche de «durabilité ». En effet, une telle démarche nécessite l'intégration et la collaboration de toutes les parties prenantes (Gouvernements, groupe d'intérêts, entreprises, citoyens...). Or, malgré ses appels répétés, le groupe n'a pu obtenir aucune aide de la part des pouvoirs publics, notamment des responsables de la protection de l'environnement et de la gestion des déchets.

Peut-on dès lors parler de procédures efficaces de développement durable, sachant que cellesci supposent une collaboration effective et partagée de toutes les parties prenantes?

\section{LIMITES DES PROJETS DE « TOURISME DURABLE »EN TUNISIE}

Compte tenu de sa position géographique, ainsi que de ses richesses naturelles, historiques et culturelles, la Tunisie détient un potentiel significatif pour le développement du secteur touristique sur le long terme.

Néanmoins, ce potentiel ne suffit pas à assurer l'instauration d'un véritable tourisme « durable », dès lors qu'il n'existe pas d'action conjointe des acteurs publics et privés de la filière touristique. Qu'il s'agisse du gouvernement, des autorités locales, des entreprises, voire des consommateurs, toutes les parties concernées ont une part de responsabilité dans le processus d'évolution en question ${ }^{26}$.

Depuis une vingtaine d'années, la Tunisie s'est efforcée de profiter de la richesse de son potentiel et d'agir de façon à promouvoir le secteur touristique sur le long terme, notamment par la mise en place de différents programmes et projets nationaux, ou en coopération avec des organismes étrangers. Mais aujourd'hui on constate malheureusement une nette réticence des entreprises à adhérer à de tels projets. Cette réticence se manifeste et s'explique selon trois points de vue.

\section{Limites environnementales}

La Tunisie a opté pour une politique environnementale fondée sur le principe du développement durable en tant que priorité nationale; à ce titre, les managers tunisiens doivent prendre cet impératif en compte au même titre que la qualité et la productivité ${ }^{27}$.

Néanmoins, selon Madame Driss, Vice-présidente de l'UTICA: «malgré sa conviction de la nécessité d'intégrer la composante environnementale, l'entreprise reste encore réticente et n'a pas été concrètement attirée par les programmes de certification». Celle-ci justifie cette réticence par «la multitude des programmes de certification qui rendent les chefs d'entreprises perplexes et désorientés ${ }^{28} »$.

Il convient de préciser que la Tunisie souffre d'un certain manque de connaissance et de conscience en matière de «Tourisme durable », qui représente encore à ce jour un «concept

\footnotetext{
${ }^{26}$ Comité 21, Agir ensemble pour un tourisme durable.

${ }^{27}$ Selon Mme Dorra Chérif, directrice de l'Assistance aux entreprises au CITET (Centre Internationale des technologies de l'Environnement de la Tunisie), la presse, 2010, économie verte, ecolabel tunisie, Clé de voûte de l'exportation.

${ }^{28}$ Selon Mme Zohra Driss, vice-présidente de I'UTICA, Webmanagercenter.com-02 Juin 2009
} 
flou » pour la plupart des chefs d'entreprises, des professionnels, des gestionnaires et même des responsables agissant au sein des institutions concernées ${ }^{29}$. Ceci peut être expliqué par un manque de savoir faire technique et une insuffisance de communication sur le sujet.

Madame Driss propose: "d'harmoniser les programmes et les interventions auprès des entreprises, de renforcer les expertises nationales existantes, d'établir une concertation effective entre les fédérations et les professionnels ${ }^{30} »$.

De nombreuses entreprises n'adhérent pas aux projets de «tourisme durable » parce qu'elles souffrent de difficultés financières, et ce malgré les incitations financières proposées par de nombreux organismes tels que: FODEP ${ }^{31}$ (Fonds de dépollution), FOCRED ${ }^{32}$ (Crédit bancaire complémentaire), ENVIROCRED ${ }^{33}$ (Ligne de crédit environnementale), FODEC $^{34}$ (Fond de développement de la compétitivité), FNME (Fond National de Maîtrise de l'énergie)... Elles ne peuvent effectivement pas investir dans un matériel restant encore «trop coûteux $x^{35}$ », particulièrement dans le contexte économique et financier délicat qui caractérise aujourd'hui la Tunisie. Madame Driss propose à ce propos «d'assouplir les moyens et les incitations des fonds et des crédits».

D'autre part, sur le plan législatif et institutionnel, il est à noter qu'aucune loi n'oblige les hôtels à surveiller leur consommation ou à entreprendre des actions de limitation de leur consommation, ainsi les mesures d'économies des ressources en eau et en énergie sont considérées d'un point de vue essentiellement financier.

«Malheureusement, la notion de " tourisme durable " n'est pas encore entrée dans les mours des entreprises tunisiennes suite à l'inexistence des textes règlementaires ou des normes sectorielles qui les obligeraient à s'orienter vers ce phénomène. En effet, tant que les entreprises ne sont pas sûres que les concurrents vont adopter le même comportement, aucune n'accepterait investir dans ce sens ${ }^{36}{ }$.

Ainsi, les parties concernées doivent mettre en place les dispositifs nécessaires afin d'aboutir à une gestion participative impliquant à la fois l'administration centrale, les institutions locales, les populations et la société civile ${ }^{37}$.

\section{Limites sociales}

\footnotetext{
${ }^{29}$ Cette étude de recherche a rencontré des difficultés majeures pour la collecte d'une information juste et pertinente même auprès des grands responsables des organismes concernés (MEDD (Ministère de l'Environnement et du Développement durable) ou même au sein de l'ONTT (Office National du Tourisme Durable).

${ }^{30}$ Webmanagercenter.com-02 Juin 2009

${ }^{31}$ Une subvention de $20 \%$ ( $\max$ ) du coût d'investissement accordée par le FODEP pour une action de dépollution

${ }^{32}$ Des facilités sur le FOCRED pour les bénéficiaires du FODEP.

${ }^{33}$ Des conditions de crédits encourageant les projets de dépollution et de maîtrise de l'énergie

${ }^{34}$ des primes accordées pour la mise à niveau des entreprises industrielles, et aux investissements technologiques à caractère prioritaire.

${ }^{35}$ Selon le responsable commercial de hôtel « The Russelior » à Hammamet Sud.

${ }^{36}$ Selon les propos d'un directeur de maintenance, cités par Ghozzi C., 2011, quelle stratégie de développement durable dans les hôtels en Tunisie, vertigo- la revue électronique en sciences de l'environnement

${ }^{37}$ Ecotourisme et tourisme culturel durable en Tunisie: Situation actuelle et perspectives, 2004.
} 
Dans la perspective d'un développement socio-économique harmonieux du pays, il est souhaitable que les activités touristiques, entre autres, s'étendent vers l'intérieur des terres, notamment en impliquant la population locale et en incitant les touristes à découvrir des régions encore très peu visitées à l'heure actuelle.

L'amélioration de la qualité des infrastructures permettant d'attirer l'investissement à l'intérieur du pays passe vraisemblablement par l'intervention des pouvoirs publics, voire de grands groupes privés. Outre la contrainte matérielle que ceci représente, il faut également prendre en considération la contrainte immatérielle que constitue la nécessaire évolution des mentalités.

C'est ainsi que la dimension sociale du «tourisme durable » est souvent difficile à admettre pour les principaux décideurs et les tour-opérateurs qui considèrent la gestion touristique comme étant une affaire devant être traitée par des spécialistes et devant obéir à des standards très rigoureux. L'implication des populations locales doit rester selon eux un phénomène marginal.

Le chemin semble encore long et parsemé d'embuches avant de parvenir à l'instauration d'une logique de collaboration nationale. Ce n'est en effet qu'au prix d'une forte évolution des mentalités que la Tunisie pourra donner toute la mesure de son potentiel touristique et économique.

\section{Limites économiques}

La Tunisie souffre actuellement d'une conjoncture difficile liée au «printemps arabe », expliquant au moins en partie le recul des investissements touristiques ${ }^{38}$ et la faible rentabilité du secteur. Les entreprises concernées sont ainsi menacées par le surendettement, tandis que les banques sont de plus en plus réticentes à leur égard. Cette situation risque d'affecter fortement et durablement l'image de la Tunisie à l'étranger. De plus, les hôteliers seront vraisemblablement tentés de réduire la qualité de leurs services, selon une logique de réduction des coûts, ce qui représente un frein à toute reprise ultérieure.

Le tourisme tunisien reste trop concentré sur le littoral; selon Chapoutot (2008), et ce pays n'a pas su valoriser ses territoires. Les tentatives de diversification des produits touristiques et de développement du tourisme à l'intérieur des terres par la promotion du tourisme saharien et du tourisme culturel n'ont pas suffi à créer un véritable équilibre en la matière. Selon le même auteur, ces formes de tourisme représentent une activité complémentaire au tourisme balnéaire, mais ne favorisent en rien la diversification économique locale, ni «l'étalement de la saison ».

Le tourisme golfique joue aussi un rôle important permettant une certaine forme de diversification. Néanmoins ce type de tourisme en Tunisie souffre d'un problème majeur lié au manque de dessertes aériennes ${ }^{39}$. Tourismag a montré après une rencontre avec les

\footnotetext{
${ }^{38}$ Les IDE en baisse en 2011 de près de 32\% par rapport à 2010. (http://www.webmanagercenter.com/actualite/economie/2011/12/15/113828/tunisie-les-ide-en-baisse-depres-de-32). I'année 2010 note elle-même un recul notable d'investissements (selon le rapport de MEDD et de I'ANPE, 2010, Le tourisme durable en Tunisie) au cours du Xème plan de mise à niveau.

39 Tourismag, 2011, Tourisme golfique en Tunisie: Packages intéressants au salon de Paris, mais...du 26 Mars 2011.
} 
professionnels que «la Tunisie a perdu le marché scandinave, qui était le premier émetteur de golfeurs vers le pays».

\section{PERSPECTIVES DU « TOURISME DURABLE » EN TUNISIE}

Malgré ces contraintes et difficultés qui entravent la mise en œuvre d'une stratégie pertinente de «tourisme durable », les responsables conscients de ses avantages, restent optimistes et travaillent actuellement sur de nombreux projets dans ce domaine.

Après consultation des principaux partenaires concernés et sur la base de la lecture et de l'analyse des principaux plans et programmes de développement en cours dans le pays, les perspectives du secteur touristique en Tunisie à horizon $2016^{40}$ restent encourageantes.

\section{Perspectives environnementales}

Le pari en cours consiste à poursuivre l'instauration d'une consommation et une production durables, à optimiser les ressources naturelles sur le long terme, à gérer harmonieusement et durablement le littoral, à rationaliser la consommation énergétique et à promouvoir les renouvelables.

On peut attendre des retombées favorables de l'écolabel Tunisien en termes de valorisation des produits et services nationaux sur les marchés étrangers ${ }^{41}$. Le projet d'écotourisme présente lui aussi des perspectives intéressantes en tant qu'ensemble d'activités liées à un site naturel et combiné à des services touristiques (hébergement, restauration, loisirs, information...). L'écotourisme consiste notamment à impliquer les populations locales et a ainsi des retombées sur leur bien être socio-économique tout en préservant l'environnement. Plusieurs autres projets environnementaux ont été lancés, dont on peut noter les exemples ciaprès. Les zones touristiques réparties le long du littoral tunisien ont été conçues selon un modèle de développement classique, sous forme de «zoning » et de stations intégrées; elles se présentent sous une forme linéaire d'aménagement, occupant de larges surfaces. Prenant la mesure des avantages et des inconvénients de cette situation, l'Etat tunisien a préparé un projet de protection des zones touristiques représentant un potentiel économique important et d'adaptation de ces zones aux changements climatiques de 3MDT étalé sur 2 ans destiné à toutes les zones touristiques.

Un autre projet concerne le lancement de l'hôtel écologique de $3 \mathrm{MDT}^{42}$ étalé sur 5 ans et destiné aux régions climato-touristiques les plus vulnérables aux changements climatiques (encore à identifier) afin de faire face à la consommation excessive d'énergie et au gaspillage de l'eau potable.

Un projet de réhabilitation du littoral touristique tunisien concernant notamment l'île de Jerba de $3 \mathrm{MDT}$ étalé sur 5 ans est prévu pour faire face à l'érosion marine et à la hausse du niveau de la mer.

\footnotetext{
${ }^{40}$ République tunisienne, Ministère du tourisme, consultation nationale, 2010, Stratégie 2016.

${ }^{41}$ Webmanagercenter.com-02 Juin 2009

${ }^{42} 1 \mathrm{MDT}$ pour l'étude et $2 \mathrm{MDT}$ pour la subvention à la construction.
} 
Un projet de gestion des ressources en eau par le secteur touristique a aussi été lancé; celui-ci comporte la réalisation d'une mini-station de dessalement de l'eau de mer pour 2000 lits en utilisant des énergies renouvelables. Le coût de ce projet est estimé à 3MDT étalé sur 3 ans et concerne aussi la zone touristique de Jerba pour faire face à la consommation de l'eau qui augmente sensiblement sous les climats secs et ensoleillés (la consommation d'eau d'un touriste représente en moyenne 9 fois la consommation d'un autochtone).

Enfin, il faut noter le lancement d'un projet de 3.5MDT étalé sur 3 ans est destiné à la même zone touristique avec comme objectif le développement de nouveaux produits touristiques, autres que ceux du tourisme balnéaire, consistant notamment à définir des régions climatotouristiques et à y créer des circuits éco-touristiques.

\section{Perspectives sociales}

Après plus de cinquante ans d'indépendance, la Tunisie souffre d'importants déséquilibres régionaux. Aujourd'hui, plusieurs plans ont été mis en place pour une allocation optimale des richesses, afin de favoriser un certain équilibre social. Dans une logique de pérennité, cet équilibre social entre les différentes régions du pays doit permettre le relèvement du niveau de développement de la société toute entière et l'instauration d'un cadre général d'intégration de l'ensemble des citoyens aux projets nationaux ${ }^{43}$.

\section{Perspectives économiques}

Pour pouvoir se repositionner favorablement, les produits touristiques tunisiens doivent et être diversifiés et de bonne qualité. Pour ce faire, la stratégie du gouvernement à l'horizon 2016 comporte un plan d'action ainsi qu'une charte de qualité appelée «Charte Jasmin » qui vise à améliorer la qualité de l'offre existante, encourager l'innovation, réaménager de nombreuses zones touristiques, diversifier les types d'hébergements touristiques («hébergement alternatif $»$ : maisons d'hôte, camping...) et inciter au développement de ces nouveaux logements.

\section{CONCLUSION}

La préoccupation de l'Etat tunisien dès 1950 a été de favoriser la rentabilité, vaincre les réticences et inciter l'investissement dans le secteur touristique, sans se préoccuper des éventuels méfaits engendrés par ce secteur. Ce n'est que dans les années 90 qu'apparait une volonté d'élaborer et d'adopter en Tunisie un cadre de «développement durable et de partenariat». Depuis lors, les plans de MAN et les programmes de certification se sont succédés, notamment le programme tuniso-allemand pour l'environnement, le programme Environnement et Energie, le projet de production propre pour la Tunisie (PPPT) et l'écolabel tunisien. Ces programmes, visant à renforcer la compétitivité des produits touristiques tunisiens, ont été initiés avec un soin particulier concernant la protection de l'environnement, dont notamment l'utilisation de techniques de production "propres », ainsi que la mise en œuvre d'actions de dépollution chaque fois que cela s'est avéré nécessaire.

\footnotetext{
43 République tunisienne, ministère de l'agriculture et de l'environnement, Direction Générale du développement durable, 2011, stratégie nationale de développement durable 2012-2016.
} 
La démarche engagée par le Groupe TUI s'inscrit parfaitement dans le cadre de cette stratégie nationale. Les succès remarquables du Groupe, et en particulier de la chaîne Magic Life, en matière de développement durable sont d'abord représentés par l'obtention des certifications ISO 14001, ISO 9001, ISO 22000, Ecolabel clef Verte et écolabel tunisien, puis par le lancement de nouveaux labels par le Groupe TUI lui-même: TUI Ecoresort et TUI Umwelt Champion.

La Direction Générale du Groupe s'est effectivement conformée au cours des dernières années au principe qu'elle a elle-même proposé, selon lequel: «le respect de l'environnement et des équilibres naturels aujourd'hui est la base du développement social, humain et écologique de demain ». Elle s'est efforcé d'impliquer toutes les parties prenantes, dans le Groupe TUI et à l'extérieur de celui-ci, dans sa démarche d'amélioration continue de la qualité en général, et plus particulièrement de la qualité des denrées alimentaire et de la protection de l'environnement.

Le groupe a suivi une stratégie de développement qu'il convient de qualifier de «vivable », dans le sens où celle-ci tient compte des facteurs environnementaux et sociaux, et où elle a permis l'élaboration d'une offre touristique dans laquelle les dimensions humaine et culturelle sont prépondérantes.

Malgré des résultats probants, le Groupe est aujourd'hui encore confronté à d'importantes difficultés liées à son implantation. Ses activités étant concentrées sur le territoire tunisien, il ne parvient pas à obtenir le soutien qu'il est en droit d'attendre des pouvoirs publics, notamment des responsables de la protection de l'environnement et de la gestion des déchets. Or il est de plus en plus évident qu'une démarche de développement durable doit pouvoir s'appuyer sur l'ensemble des acteurs socio-économiques présents (Gouvernement, entreprises, citoyens, groupe d'intérêts) pour être véritablement efficace.

Depuis une vingtaine d'années, les autorités tunisiennes se sont efforcées de valoriser le potentiel économique du pays, notamment grâce au développement du secteur touristique, en suivant une politique de grands projets d'aménagement et de coopération avec des organismes institutionnels étrangers. Malheureusement, cette politique qui aurait dû aboutir à l'avènement d'un véritable «tourisme durable » en Tunisie est aujourd'hui entravée par les réticences des entreprises locales à l'égard des projets en cours. Il semble néanmoins que leurs réticences soient moins liées à leurs convictions en matière de protection de l'environnement qu'à des considérations d'ordre économique.

C'est effectivement dans un contexte socioéconomique difficile que certains responsables du secteur public comme du secteur privé s'efforcent de relancer la concertation sur le « tourisme durable » au niveau national. De façon officielle, la politique du Gouvernement tunisien intègre cette préoccupation qui apparaît toujours dans les plans et les programmes de développement procédant de la «Stratégie du tourisme à horizon 2016 ».

Cette volonté nationale va devoir s'affirmer face aux problèmes d'image qu'engendre le «printemps arabe », à une conjoncture économique globalement défavorable, aux difficultés financières des entreprises tunisiennes et aux réticences de tous ordres. La marche vers la « durabilité » est loin d'être achevée et ne pourra vraisemblablement se poursuivre qu'au prix d'une entente et d'une collaboration de toutes les parties prenantes au niveau national. 
L'évolution des mentalités est ainsi probablement le chantier le plus important à mettre en œuvre dans la perspective d'une prospérité fondée sur le respect des personnes et de l'environnement.

\section{BIBLIOGRAPHIE}

Ayerbe, C., Missonier, A. (2007), "Validité interne et validité externe de l'étude de cas: principes et mise en œuvre pour un renforcement mutuel", Finance Contrôle Stratégie, Vol. 10, N², juin, pp. 37-62

Bernhard, C. (2007), "Les contraintes environnementales, frein ou atout pour le développement local ? ", Veille info tourisme.

Camus, S., Hikkerova L., Sahut JM. (2010), "Tourisme durable : une approche systémique" Management et Avenir, $\mathrm{N}^{\circ} 34$, Avril.

Chaieb, M.S (2009), "Vers des alternatives au zoning touristique en Tunisie", Magazine d'architecture en ligne, Arch-Mag, http://www.archi-mag.com/essai_42.php.

Chand, M. (2006), "The Relationship between Corporate Social Performance and Corporate Financial Performance: Industry Type as a Boundary Condition", The Business Review, vol. 5, $\mathrm{N}^{\circ} 1$, pp 240-245.

Chapoutot, JM (2008), "Suivi de la stratégie méditerranéenne pour le développement durable", Plan Bleu, Centre d'Activités Régionales, Sophia-Antipolis, Juin.

Cromer, G. (2011), "Tourisme durable: Quelles garanties?", Juristourisme, n ${ }^{\circ} 131$, Mai.

Da Luz, J. (2008), "Durable, équitable, responsable...un Challenge Tourisme pour Madagascar", Tourmag, 16 Mai.

Decock-Good, C. (2001), "L'engagement mécanique des entreprises: mesure de l'une des expressions de leur responsabilité sociétale", Finance Contrôle Stratégie, 4, pp. 29-57.

Gallouj, C. (2011), "Résultats et perspectives des recherches en tourisme et développement durable: une discussion à partir des travaux français", $2^{\text {ème }}$ journées scientifiques du Tourisme Durable, IPAG, Poitiers, 23 juin.

Germain, K. (2011), "Des hôtels qui ont pris le virage durable", Chaire de tourisme transat.

Hergli, S., Sahut JM. et Teulon F. (2012), "Politiques et stratégies de développement durable dans le secteur hôtelier : le cas d'ACCOR", Management et Avenir, N56, Sept.

Leroux, E. (2010), "Tourisme durable et écotourisme: des méthodes de calcul liées au marketing", Gestion 2000, $\mathrm{n}^{\circ} 1$.

Margolis, JD, Elfenbein, HA and Walsh, JP. (2008), "Do Well by Doing Good? Don't Count on It", Harvard Business Review, vol. 86, $\mathrm{n}^{\circ} 1$.

Merasli, S. (2004), "La qualité dans l'industrie du tourisme: un impératif majeur pour un tourisme durable", Théoros, 23-2.

Parra, C, Moulaert, F. (2011), "La nature de la durabilité sociale: Vers une lecture socioculturelle du développement territorial durable", Développement durable et territoires, Vol. 2, $\mathrm{N}^{\circ} 2$, Mai.

Peters Van Deinse, S. (1999), "Nouvelles normes ISO", L'usine Nouvelle, n²688, Mai.

Schéou, B. (2009), Du tourisme durable au tourisme équitable: quelle éthique pour le tourisme de demain? , Les métiers Tourisme, De Boeck

Van Beurden, P and Gössling, T. (2008), "The worth of values - A literature review on the relation between corporate social and financial performance", Journal of Business Ethics, Vol. 82, n² 2 , pp. 407-424.

WCED (1987), "Our common future," The Brundtland Report, Oxford University Press, Oxford: England, 1987. 
